BMJ Open

Diabetes

Research

\& Care

\section{The relationship of individual comorbid chronic conditions to diabetes care quality}

To cite: Magnan EM, Palta M, Mahoney JE, et al. The relationship of individual comorbid chronic conditions to diabetes care quality. BMJ Open Diabetes Research and Care 2015;3: 0000080 . doi:10.1136/bmjdrc-2015000080

Received 6 January 2015 Revised 16 April 2015 Accepted 13 May 2015

\section{CrossMark}

For numbered affiliations see end of article.

\section{Correspondence to} Dr Elizabeth M Magnan; elizabeth.magnan@ucdmc. ucdavis.edu

\author{
Elizabeth M Magnan, ${ }^{1,2}$ Mari Palta, ${ }^{3,4}$ Jane E Mahoney, ${ }^{5}$ Nancy Pandhi, ${ }^{2,6}$ \\ Daniel M Bolt, ${ }^{7}$ Jennifer Fink, ${ }^{8,9,10}$ Robert T Greenlee, ${ }^{11}$ Maureen A Smith ${ }^{2,4,6,12}$
}

\section{ABSTRACT}

Objective: Multimorbidity affects 26 million persons with diabetes, and care for comorbid chronic conditions may impact diabetes care quality. The aim of this study was to determine which chronic conditions were related to lack of achievement or achievement of diabetes care quality goals to determine potential targets for future interventions.

Research design and methods: This is an exploratory retrospective analysis of electronic health record data for 23430 adults, aged 18-75, with diabetes who were seen at seven Midwestern US health systems. The main outcome measures were achievement of six diabetes quality metrics in the reporting year, 2011 (glycated haemoglobin (HbA1c) control and testing, low-density lipoprotein control and testing, blood pressure control, kidney testing). Explanatory variables were 62 chronic condition indicators. Analyses were adjusted for baseline patient sociodemographic and healthcare utilization factors. Results: The 62 chronic conditions varied in their relationships to diabetes care goal achievement for specific care goals. Congestive heart failure was related to lack of achievement of cholesterol management goals. Obesity was related to lack of $\mathrm{HbA1C}$ and BP control. Mental health conditions were related to both lack of achievement and achievement of different care goals. Three conditions were related to lack of cholesterol testing, including congestive heart failure and substance-use disorders. Of 17 conditions related to achieving control goals, 16 were related to achieving $\mathrm{HbA1C}$ control. One-half of the comorbid conditions did not predict diabetes care quality.

Conclusions: Future interventions could target patients at risk for not achieving diabetes care for specific care goals based on their individual comorbidities.

The vast majority, over $83 \%$, of patients with diabetes in the USA, has at least one other co-occurring chronic condition, and diabetes care quality remains suboptimal with staggering levels of morbidity and mortality. ${ }^{1}{ }^{2}$ Multimorbidity adds additional care demands to the already complex diabetes care. The US Department of Health and

\section{Key messages}

- Individual conditions have unique relationships with diabetes care goal achievement.

- Congestive heart failure was associated with lack of achievement of both low-density lipoprotein control and testing goals.

- Individual mental health conditions were associated with not achieving certain care goals, and achieving other care goals.

\section{Future research questions}

- Do these relationships hold up in other populations?

- How do individual conditions and combinations of individual conditions relate to other outcomes in patients with diabetes?

- How can we best improve care for patients with diabetes and select comorbid conditions?

Human Services, The Institute of Medicine, the Patient-Centered Outcome Research Institute and others have recognized the need to understand and improve multimorbidity care. ${ }^{3-6}$ Unfortunately, there are few current multimorbidity interventions to improve diabetes care, as demonstrated by a recent Cochrane review. ${ }^{7}$ One reason for the limited number of interventions may be that the relationship of individual comorbid chronic conditions to elements of diabetes care is not known. ${ }^{3}$ Only a few specific conditions, such as hypertension and depression, ${ }^{8-10}$ have been assessed for their individual impact on diabetes care and even then for a limited set of care goals. As diabetes care quality consists of achieving multiple care goals, including both testing and control goals, individual conditions could have a different relationship with diabetes care for each care goal. Without knowing these relationships, we cannot identify patients at risk for not achieving care goals 
based on their comorbidities, and we cannot target interventions to improve care for specific diabetes care goals in patients with the selected comorbidities.

A conceptual model suggests that the relationship between comorbid conditions and diabetes care is due to characteristics of the comorbid condition. ${ }^{11}$ In this framework, patients with conditions that share care goals with diabetes (concordant) are more likely to achieve diabetes care goals, while patients with conditions that do not share care (discordant) are less likely to achieve diabetes care goals. In addition, care for severe and/or symptomatic conditions would preferentially be prioritized over care for diabetes. Past studies on the impact of chronic conditions on diabetes management have considered only one condition characteristic (eg, concordant/discordant or severe/not severe) and considered all conditions classified as the same type (eg, discordant) as equivalent when determining impact on diabetes care. ${ }^{11-16}$ However, in actuality, some conditions might have a greater or lesser impact on diabetes testing and control goal achievement than other conditions. For example, a diagnosis of cancer might have a greater impact on diabetes management than heartburn. It is most likely that the overall impact of a chronic condition on diabetes care is due to a combination of its many characteristics (concordant/discordant, severity, symptom level) as well as other factors such as treatment needs and pathophysiology. Therefore, it is important to consider the impact of individual conditions, not just classes of conditions based on their characteristics.

The objective of this study was to determine the relationship between individual chronic conditions and the achievement of publicly-reported diabetes quality care goals in patients with diabetes. We did this at the individual condition level to study the combined impact of all potential condition characteristics. We hypothesized that the relationship between the condition and the achievement of diabetes care goals would vary for each condition and care goal, with achievement of that goal (positive association with achievement), lack of achievement of that goal (negative association with achievement), or no relationship with care (no association).

\section{RESEARCH DESIGN AND METHODS \\ Design and setting}

We conducted a retrospective analysis of 2 years of electronic health data, 2010 (baseline year) and 2011 (quality reporting year), for seven health systems that participated in a Midwestern quality reporting collaborative, the Wisconsin Collaborative for Healthcare Quality. The health systems are academic and community practices in rural, suburban and urban settings, and all submit data electronically for public reporting using standardized methodology. ${ }^{17}$ The Minimal Risk Health Sciences Institutional Review Board at the University of Wisconsin determined that the project was exempt from oversight.

\section{Patients}

All patients with a diagnosis of diabetes (type 1 or type 2 ), who were 18-75 years old, and were medically homed within the seven participating health systems were included in the sample. ${ }^{17}$ We focused on patients eligible for public reporting of diabetes quality care metrics to ensure general consensus about the definition of diabetes quality care goals. Diabetes was defined by at least two face-to-face ambulatory visits (using CPT-4 outpatient evaluation and management codes) with any clinician with an International Classification of Diseases Ninth Edition (ICD-9) diagnosis code of 250.XX, 357.2, 362.0X, 366.41 or $648.0 \mathrm{X}$ on different dates of service over the 2 years of data. ${ }^{17}$ Medically homed was defined as at least two face-to-face office visits to the group on different dates of service in the past 2 years, with at least one visit in the reporting year. ${ }^{17}$

\section{Primary outcome variables}

Our primary outcome variables were the achievement of three control and three testing diabetes care goals, recommended in clinical guidelines, used in public reporting, measurable in a standardized fashion across populations and health systems and shown to be associated with macrovascular and microvascular outcomes in diabetes. ${ }^{1718}$ Variables were binary, representing care goal achievement or not in the reporting year, as defined by the public reporting collaborative that reports on the participating health systems. The three control goals were: HbAlc control $<7 \%$ (or $<8 \%$ if $65-75$ years old or having specific comorbidities) $;{ }^{17-19}$ LDL cholesterol control $<100 \mathrm{mg} / \mathrm{dL}$; blood pressure (BP) control at $<130 / 80 \mathrm{~mm} \mathrm{Hg}$. The specific comorbidities were: coronary artery bypass graft, percutaneous transluminal coronary angioplasty, ischemic vascular disease, congestive heart failure, myocardial infarction, chronic renal failure, dementia, blindness and amputation (lower extremity). ${ }^{17}$

The three testing goals measured in the reporting year were as follows: HbAlc testing two or more times; LDL cholesterol testing; kidney function testing by either a urine microalbumin test or documented evidence of nephropathy. ${ }^{17} 18$ Documented evidence of nephropathy, as defined by the public reporting collaborative that reports on the participating health systems, included a visit to a nephrologist for any reason, a diagnosis code for chronic kidney disease or renal manifestations of diabetes or evidence of dialysis treatment during the reporting year. ${ }^{17}$

\section{Main explanatory variables}

Indicator variables for patient comorbidities were the main explanatory variables. We used a comprehensive set of 62 chronic condition indicators (excluding diabetes) based on an established list of outpatient-relevant chronic conditions developed from the AHRQ Clinical Classification Software (CCS) categories for medical conditions. ${ }^{20}{ }^{21}$ The 62 chronic condition indicators cover 
1412 ICD-9 codes, with several codes for similar or the same condition(s) counted as the presence of one condition (eg, multiple codes for types of coronary artery disease are counted as the patient having a coronary artery disease). Chronic conditions were defined as ICD-9 codes billed at one or more face-to-face visits in the baseline year. This was carried out to ensure that the conditions were present and actively managed in the clinic before the quality reporting time frame. See online supplementary appendix table 1 for a list of the 62 conditions organized by body system.

\section{Covariates}

We defined variables for patient sociodemographic characteristics and healthcare utilization. Sociodemographic characteristics were age (continuous), gender, race (white or other) and insurance (non-commercial Medicare, Medicaid, commercial (including commercial Medicare) or self-pay/unreported). We defined two additional socioeconomic status proxy variables by linking patient zip codes to census tract data. These are the per cent of the population in the patient's zip code who live below the poverty line and the per cent who do not have a high school education. Healthcare utilization was defined as number of face-to-face office visits in the baseline year (coded as 4 categories). ${ }^{1722}$ We also included an indicator for health system to account for clustering. To account for potential contextual effects, we developed a variable for the prevalence of self-reported diabetes in each patient's county of residence, and a variable for the per cent of all Medicare patients in the county who have had HbAlc testing, from patient zip codes linked to the University of Wisconsin Population Health Institute-Robert Wood Johnson County Health Rankings. ${ }^{23}$ We also determined rural-urban commuting area codes based on each patient's zip code (RUCA, 4 level) ${ }^{24}$

\section{Statistical analyses}

All analyses were conducted using Stata V.13.0 (StataCorp, College Station, Texas, USA). There were no missing data for any covariates or outcome variables. Outcome variables were coded as testing or control thresholds being met when in the health record, and not being met when not recorded, as is the standard for public reporting. Descriptive analyses summarized categorical variables using percentages and continuous variables using means with SDs. Logistic regression models were fit for each of the six diabetes control and testing goals, to assess the relationship between 58 individual chronic conditions and the achievement of each care goal, adjusted for covariates as described above. All conditions with more than 20 patients and all covariates were used in all models to include all potentially relevant variables. Four conditions with fewer than 20 conditions $(<0.1 \%$ prevalence) were not included in the model: cystic fibrosis, tuberculosis, sickle cell anemia and amyloidosis. Results are reported as OR and 95\% CIs. The significance of each condition on diabetes goal achievement was determined at $\mathrm{p}$ value $<0.01$. These conservative significance values were used for this exploratory study to account for multiple comparisons, while avoiding unnecessary increases in type 2 error that can occur with Bonferroni correction. All conditions with significant associations ( $p$ value $<0.01$ ) were present in at least 93 patients.

\section{RESULTS}

\section{Patient characteristics}

Our sample had 23430 patients with diabetes, between the ages of 18 and 75 (table 1). Patients had 0-22 comorbid conditions, with an average of $3.8(\mathrm{SD}=2.5)$ and a median of 3 . The frequencies of all 62 chronic conditions ranged from $77 \%$ for hyperlipidemia to $0.01 \%$ for tuberculosis (see online supplementary appendix table 1).

\section{Relationship of individual chronic conditions to diabetes control}

After controlling for patient sociodemographic factors and number of office visits, nine conditions predicted lack of achievement of specific control goals (table 2). Of these conditions, five were related to not achieving HbA1c control, including obesity and depression. Eight conditions were related to lack of achievement of cardiovascular risk reduction goals (LDL and/or BP control). These included: congestive heart failure (CHF), hypertension, obesity, anxiety and substance abuse.

Seventeen conditions were related to achieving control goals, and 15 to HbAlc control. Among these were schizophrenia and bipolar disorder. Blood pressure control was achieved with four conditions, including obesity and substance use disorders.

\section{Relationship of individual chronic conditions to diabetes testing}

All conditions that were related to lack of achievement of testing goals were related to LDL testing (table 3). These included: CHF and substance use disorders.

Obesity was among six conditions related to achieving the HbAlc testing goal. Renal failure was strongly associated with achieving kidney testing with an OR of 29.1 (95\% CI 18.6 to 45.4 ); a diagnosis of renal failure meets the kidney testing metric by definition.

\section{Conditions with no relationship to diabetes control or testing}

Of 62 conditions, 33 were related to neither control nor testing care goal achievement at $\mathrm{p}$ value $<0.01$, 4 additional conditions were not related to control but were related to testing, and 17 were not related to testing but were related to control (table 4). These conditions span multiple organ systems including cardiovascular and mental health conditions, and many are low prevalence. 
Total sample $(n=23$ 430)

\section{Comorbidities}

None (diabetes only, no other chronic conditions), \% $\quad 7.7$

Number of total comorbid conditions, mean (SD)

Number of concordant comorbid conditions, mean (SD)

$2.2(1.3)$

Number of discordant comorbid conditions, mean (SD)

$1.7(1.7)$

Age, mean (SD)

Sex, female, \%

Race/ethnicity, white, \%

Insurance, \%

Commercial

70

Medicare

Medicaid

Uninsured/unreported, \%

Number of visits in baseline year

$<2$

RUCA, by patient's zip code, \%

Urban core

Suburban

$\%$, percentage; HbA1c, glycated haemoglobin.

\section{DISCUSSION}

We found that, as expected, the 62 chronic conditions varied in their relationships to diabetes care goal achievement for specific care goals. Several of the results are especially pertinent to diabetes care and the development of future interventions for care in multimorbidity. CHF, obesity, and mental health disorders were all related to lack of achievement for specific goals. HbAlc control was achieved across multiple conditions, and for many more conditions than LDL or BP control. Lack of achievement of a testing goal was only seen with LDL testing, with three individual conditions. Finally, half of the comorbid conditions had no relationship to any of the six diabetes care goals. Future interventions can target selectively patients with specific comorbidities that are associated with lack of achievement of diabetes care goals.

CHF was related to lack of achievement of both LDL control and testing. This was surprising as LDL management is integral in CHF care, and based on the conceptual model of shared care leading to better diabetes care, we expected CHF to be associated with achieving LDL management goals. ${ }^{1125} \mathrm{CHF}$ showed no significant association with BP control or HbAlc testing or control. The reason that patients with CHF appear less likely to achieve LDL control, but not less likely to achieve BP control, an important goal in $\mathrm{CHF}^{25}$ or HbAlc control, could be due in part to patient priorities as a past study that showed that cholesterol control was a lower priority than $\mathrm{BP}$ or $\mathrm{HbAlc}$ control to patients with diabetes. ${ }^{26}$

Obesity was related to lack of achievement of HbAlc and BP control, but achievement of the HbAlc testing goals. The lack of control could be due to glucose and BP dysregulation in obesity, or lifestyle factors that worsen weight, HbAlc, and BP control. The lack of HbAlc control despite guideline-adequate testing highlights the relative difficulty of achieving control compared to completing a non-fasting laboratory test. Control requires counseling at a visit and between-visit self-care for a guideline-recommended $2 \mathrm{~h} /$ day, ${ }^{27}$ while ordering a laboratory test and completing it at the visit, requires relatively minimal effort from the provider and patient.

Interestingly, while three mental health conditions were associated with not achieving diabetes care for specific care goals, this was not for the same goals, and not all mental health conditions were related to lack of care goal achievement. Mental health conditions can present barriers to diabetes care through lack of motivation or an ability to perform self-care. ${ }^{82}$ Patients who do not take prescribed medications or follow lifestyle recommendations are less likely to achieve diabetes care goals. Although we found associations with lack of care goals achievement for patients with depression, substance 
Table 2 Impact of individual concordant and discordant conditions on diabetes control goal achievement, adjusted, OR (95\% Cl), with prevalence of conditions, among patients with diabetes age $18-75$

\begin{tabular}{|c|c|c|c|c|c|c|c|}
\hline & \multirow{2}{*}{$\begin{array}{l}\text { Prevalence \% } \\
(n=23430)\end{array}$} & \multicolumn{2}{|l|}{ HgbA1c control } & \multicolumn{2}{|l|}{ LDL control } & \multicolumn{2}{|l|}{ BP control } \\
\hline & & OR (95\% Cl) & p Value & OR (95\% Cl) & p Value & OR (95\% Cl) & p Value \\
\hline \multicolumn{8}{|l|}{ Lack of control goal achievement } \\
\hline Obesity & 22 & 0.9 (0.8 to 0.97$)$ & 0.0060 & & & 0.9 (0.85 to 0.97$)$ & 0.0050 \\
\hline Depression & 15 & $0.9(0.8$ to 0.9$)$ & 0.0010 & & & & \\
\hline Anxiety disorders & 8.0 & & & 0.8 (0.8 to 0.9$)$ & 0.0010 & & \\
\hline CNS/PNS disorders* & 7.8 & $0.8(0.7$ to 0.9$)$ & 0.0000 & & & & \\
\hline Congestive heart failure & 5.0 & & & $0.8(0.7$ to 0.9$)$ & 0.0060 & & \\
\hline Skin ulcer & 4.8 & $0.8(0.7$ to 0.9$)$ & 0.0010 & & & & \\
\hline Degenerative eye problem & 4.3 & $0.7(0.6$ to 0.8$)$ & 0.0000 & & & & \\
\hline Substance use disorders & 0.8 & & & & & $0.6(0.43$ to 0.8$)$ & 0.0010 \\
\hline \multicolumn{8}{|l|}{ Mixed lack of achievement and achievement } \\
\hline Hypertension & 74 & 1.1 (1.04 to 1.2$)$ & 0.0030 & $1.2(1.1$ to 1.3$)$ & 0.0000 & $0.6(0.55$ to 0.6$)$ & 0.0000 \\
\hline \multicolumn{8}{|l|}{ Control goal achievement } \\
\hline Hyperlipidemia & 77 & $1.1(1.1$ to 1.2$)$ & 0.0000 & $1.3(1.2$ to 1.3$)$ & 0.0000 & $1.3(1.17$ to 1.3$)$ & 0.0000 \\
\hline Osteoarthritis & 16 & $1.2(1.1$ to 1.3$)$ & 0.0000 & & & & \\
\hline Coronary atherosclerosis & 14 & $1.3(1.2$ to 1.4$)$ & 0.0000 & $1.5(1.4$ to 1.6$)$ & 0.0000 & $1.3(1.21$ to 1.4$)$ & 0.0000 \\
\hline Allergic rhinitis & 11 & $1.2(1.1$ to 1.3$)$ & 0.0000 & & & & \\
\hline Cancer & 7.6 & $1.3(1.1$ to 1.4$)$ & 0.0000 & & & & \\
\hline Cardiac dysrhythmia & 7.3 & & & & & $1.2(1.05$ to 1.3$)$ & 0.0040 \\
\hline Kidney and vesicoureteral disorders $†$ & 4.8 & & & $1.2(1.05$ to 1.4$)$ & 0.0090 & & \\
\hline Gout or other crystal arthropathy & 4.4 & $1.2(1.1$ to 1.4$)$ & 0.0050 & & & & \\
\hline Benign prostatic hypertrophy & 4.1 & $1.3(1.1$ to 1.6$)$ & 0.0010 & $1.2(1.05$ to 1.4$)$ & 0.0090 & & \\
\hline Bipolar disorder & 3.7 & $1.2(1.1$ to 1.4$)$ & 0.0090 & & & & \\
\hline Rheumatoid arthritis & 1.5 & $1.6(1.3$ to 2.1$)$ & 0.0000 & & & & \\
\hline Schizophrenia and psychotic disorders & 1.1 & 1.7 (1.2 to 2.2$)$ & 0.0010 & & & & \\
\hline Aneurysm & 0.7 & $1.9(1.2$ to 3$)$ & 0.0060 & & & & \\
\hline Epilepsy & 0.7 & 2.1 (1.4 to 3.1$)$ & 0.0000 & & & & \\
\hline Myocardial infarction§ & 0.5 & 2.0 (1.2 to 3.2$)$ & 0.0080 & & & & \\
\hline Parkinson's disease & 0.4 & $2.8(1.4$ to 5.4$)$ & 0.0020 & & & $2.4(1.47$ to 3.9$)$ & 0.0000 \\
\hline \multicolumn{8}{|c|}{$\begin{array}{l}\text { Adjusted for age, sex, race, insurance, number of face-to-face office visits in the baseline year, pat } \\
\text { Medicare patients with diabetes in patient's county who achieved HbA1c testing, per cent below pc } \\
\text { health system. } \\
\text { All } 62 \text { conditions were included in the model. Only conditions with significant associations at p valu } \\
\text { `Excludes malignancies, multiple sclerosis and epilepsy. } \\
\text { †Excludes kidney failure. } \\
\text { §Within past } 2 \text { years. } \\
\text { CNS/PNS, central nervous system and peripheral nervous system; HbA1c, glycated haemoglobin. }\end{array}$} \\
\hline
\end{tabular}


Table 3 Impact of individual concordant and discordant conditions on diabetes testing goal achievement, adjusted, OR (95\% Cl), with prevalence of conditions, among patients with diabetes age $18-75$

\begin{tabular}{|c|c|c|c|c|c|c|c|}
\hline & \multirow{2}{*}{$\begin{array}{l}\text { Prevalence \% } \\
(n=23 \text { 430) }\end{array}$} & \multicolumn{6}{|c|}{ Diabetes care goal achieved } \\
\hline & & \multicolumn{2}{|c|}{ HbA1c testing } & \multicolumn{2}{|l|}{ LDL testing } & \multicolumn{2}{|l|}{ Kidney testing } \\
\hline \multicolumn{8}{|l|}{ Lack of testing goal achievement } \\
\hline Substance use disorders & 0.8 & & & $0.6(0.4$ to 0.8$)$ & 0.0010 & & \\
\hline Anemia & 0.4 & & & $0.4(0.2$ to 0.7$)$ & 0.0020 & & \\
\hline \multicolumn{8}{|l|}{ Testing goal achievement } \\
\hline Hyperlipidemia & 77 & $1.4(1.3$ to 1.5$)$ & 0.0000 & 2.9 (2.7 to 3.2$)$ & 0.0000 & $1.4(1.3$ to 1.5$)$ & 0.0000 \\
\hline Thyroid disorder & 15 & $1.2(1.1$ to 1.3$)$ & 0.0010 & & & & \\
\hline Coronary atherosclerosis & 14 & & & $1.3(1.1$ to 1.5$)$ & 0.0010 & & \\
\hline Renal failure & 11 & $1.3(1.2$ to 1.5$)$ & 0.0000 & & & 29.1 (18.6 to 45.4$)$ & 0.0000 \\
\hline CNS/PNS disorders ${ }^{*}$ & 7.8 & $1.2(1.1$ to 1.4$)$ & 0.0060 & & & & \\
\hline Kidney and vesicoureteral disorders $†$ & 4.8 & & & & & $2(1.5$ to 2.7$)$ & 0.0000 \\
\hline Menopause and perimenopause & 3.6 & & & $1.5(1.1$ to 1.9$)$ & 0.0030 & & \\
\hline
\end{tabular}

\section{Bold represents conditions associated with lack of goal achievement.}

Adjusted for age, sex, race, insurance, number of face-to-face office visits in the baseline year, patient rural-urban commuting area, per cent with diabetes in patient's county, per cent of

Medicare patients with diabetes in patient's county who achieved HbA1c testing, per cent below poverty line in patient's zip code, per cent without high school education in patient's zip code, health system.

All 62 conditions were included in the model. Only conditions with significant associations at $p$ value $<0.01$ are listed in the table. All conditions are mutually exclusive.

*Excludes malignancies, multiple sclerosis and epilepsy.

\#Excludes kidney failure.

CNS/PNS, central nervous system and peripheral nervous system; HbA1c, glycated haemoglobin 
Table 4 Individual chronic conditions with no significant effect on diabetes control or testing goal achievement

\begin{tabular}{|c|c|c|}
\hline Both control and testing goal achievement & Control goal achievement only & Testing goal achievement only \\
\hline \multirow{4}{*}{$\begin{array}{l}\text { Cardiac, vascular and pulmonary } \\
\text { COPD or asthma } \\
\text { Stroke and TIA } \\
\text { Peripheral atherosclerosis }\end{array}$} & Genitourinary and reproductive & Cardiac, vascular and pulmonary \\
\hline & Renal failure & Cardiac dysrhythmia \\
\hline & Menopause/perimenopause & Aneurysm \\
\hline & & Myocardial infarction \\
\hline Vascular disease ${ }^{*}$ & Allergy and immunity & \\
\hline Cardiomyopathy & Immunity disorder & Muscoskeletal \\
\hline Heart valve disorder & Endocrine & Osteoarthritis \\
\hline Pulmonary heart disease & Thyroid disorder & Gout \\
\hline Congenital heart disease & & Mental health \\
\hline Thrombosis and embolism & & Depression \\
\hline Muscoskeletal & & Anxiety disorders \\
\hline Back problem & & Bipolar disorder \\
\hline Mental health & & Schizophrenia \\
\hline Behavior disorders & & Genitourinary and reproductive \\
\hline Personality disorder & & Benign prostatic hypertrophy \\
\hline Sleep disorders & & Allergy and immunity \\
\hline Gastrointestinal & & Allergic rhinitis \\
\hline Pancreatitis & & Rheumatoid arthritis \\
\hline Intestinal disorders & & Hematological and oncological \\
\hline \multicolumn{2}{|l|}{ Liver disease $\dagger$} & cancer \\
\hline \multicolumn{2}{|l|}{ Esophageal disorder } & Neurological \\
\hline \multicolumn{2}{|l|}{ Hepatitis } & Epilepsy \\
\hline \multicolumn{2}{|l|}{ Genitourinary and reproductive } & Parkinson's disease \\
\hline \multicolumn{2}{|l|}{ Female genitourinary disorders } & Other \\
\hline \multicolumn{2}{|l|}{ Polycystic ovarian syndrome } & Degenerative eye disorder \\
\hline \multicolumn{2}{|l|}{ Allergy and immunity } & Skin ulcer \\
\hline \multicolumn{3}{|l|}{ HIV } \\
\hline \multicolumn{3}{|l|}{ Lupus } \\
\hline \multicolumn{3}{|l|}{ Tuberculosis } \\
\hline \multicolumn{3}{|l|}{ Hematological and oncological } \\
\hline \multicolumn{3}{|l|}{ Anemia } \\
\hline \multicolumn{3}{|l|}{ Sickle cell anemia } \\
\hline \multicolumn{3}{|l|}{ Neurological } \\
\hline \multicolumn{3}{|l|}{ Migraines } \\
\hline \multicolumn{3}{|l|}{ Dementia } \\
\hline \multicolumn{3}{|l|}{ Paralysis } \\
\hline \multicolumn{3}{|l|}{ Multiple sclerosis } \\
\hline \multicolumn{3}{|l|}{ Other } \\
\hline \multicolumn{3}{|l|}{ Non-cardiac congenital disorder } \\
\hline \multicolumn{3}{|l|}{ Sarcoidosis } \\
\hline \multicolumn{3}{|l|}{ Amyloidosis } \\
\hline Cystic fibrosis & & \\
\hline
\end{tabular}

abuse and anxiety, we also found care goals achievement with schizophrenia and bipolar disorder. Previous studies on major mental health disorders in diabetes showed better HbAlc control among those with schizophrenia than those without major mental health conditions. ${ }^{29}$ This could be due to increased provider attention on these perceived high-risk patients. Our results suggest that mental health barriers to diabetes care vary by both condition and care goal, and are more complicated than a simple negative association with any care for all mental health conditions.
Notably, the vast majority of conditions that were related to achieving at least one control goal were related to achieving HbAlc control (15 of 17), while only seven conditions were related to achieving cardiovascular control goals (LDL and $\mathrm{BP}$ ). This is a matter of concern as evidence shows that cardiovascular risk reduction is more likely to reduce mortality in diabetes than glycemic control. ${ }^{30} 31$ Several of the conditions that were related to achieving HbAlc control, but not cardiovascular risk reduction, are not traditionally considered diabetes-related (eg, allergic rhinitis, cancer) and should 
be less likely to support HbA1c control. ${ }^{11}$ These results may be at least partially explained by HbA1c control being prioritized over other care in diabetes, especially by patients, ${ }^{26}$ as glycemic control has long been the major focus on diabetes care.

All conditions that were related to any lack of achievement of testing goals were related to not achieving the LDL testing goal. One possible explanation for the lack of LDL testing with many of these conditions is the potential lack of provider cuing to order the test when LDL testing is not a shared care goal between diabetes and the comorbidity. ${ }^{11}$ We have seen in prior work that patients with diabetes and conditions that share cardiovascular care, such as hyperlipidemia, are more likely to have LDL testing performed than patients who do not have conditions that share diabetes care. ${ }^{12}$ Another possible explanation is that the LDL test is more challenging for patients to complete, compared to the HbAlc or kidney test, as it requires fasting and often a return visit. There were no conditions associated with not achieving HbAlc or kidney testing, both tests that can be performed without fasting. Past work showed patients prioritize diabetes care less than other healthcare when they had non-diabetes-related competing health problems. ${ }^{13}$ It could be that patients do not prioritize an inconvenient test when they have other competing demands that do not share the LDL testing goal. ${ }^{11-13}$

Over half of the conditions studied had no relationship to any testing or control goals. Among these conditions are many that share diabetes care goals (eg, peripheral atherosclerosis) and could be expected to support goal achievement. ${ }^{11}$ Many were conditions that can be symptomatic (eg, back problems) or severe (eg, stroke), and therefore could distract from diabetes care. ${ }^{11}$ This finding suggests that overlap of goals with diabetes care, or potential for symptoms, is alone insufficient to determine if a comorbid condition will be related to achievement or lack of achievement of diabetes care goals. Other factors, such as preference for care, patient context, and health system characteristics, play a role and must be considered in multimorbidity care. $^{5} 32$

\section{Strengths and limitations}

The major strengths of this study are the inclusion of a comprehensive set of 62 chronic conditions, six diabetes care goals, and a large sample across multiple health systems. It is important to acknowledge that the sample is from one Midwest state and only includes health systems that participate in public reporting and that this may limit generalizability. However, patients come from a wide range of ages, race/ethnicities and payors, we were able to adjust for multiple patient characteristics, the health systems did not all share any common care approaches or characteristics, and diabetes care goal achievement was similar to results from a national sample in $2011 .{ }^{33}$ While we included several sociodemographic variables, we recognize that in our study, as in any similar analysis, there were unmeasured patient characteristics for which we could not make adjustments. To establish actively managed comorbid conditions, we used ICD-9 codes billed at face-to-face visits in the baseline year, as has been performed previously. ${ }^{2021}$ There is a risk for underdiagnosis of certain conditions with this approach, such as obesity ${ }^{34}$ and depression; ${ }^{35}$ however, there is less risk of bias than with condition patient selfreport. ${ }^{21}$ There is also a potential for patients to receive some care and achieve diabetes care goals outside of their medical home health system, and this goal achievement would not be recognized in our analysis. Our statistical approach included multiple variables, and many conditions were of low prevalence. We chose not to correct for multiple comparisons, such as with the Bonferroni method, to avoid increasing type 2 error in this first-step study. Instead, we used a p value $<0.01$ to determine significant associations. We do note that the impact of some conditions on care goal achievement are small in magnitude while statistically significant (ie, OR 1.1) and their clinical significance therefore could be limited and should be tested in other populations in future work. There is also a potential for interaction effects among conditions, but these were not studied in the current analyses and the impact of combinations of conditions should be studied in future work. Finally, we used publicly reported testing and control care goals as markers of diabetes quality, as these are relevant to our population of patients with diabetes, consistently measurable, and there is agreement that achieving these goals leads to better long-term outcomes. ${ }^{7} 31$ It is important to note that there are other aspects of care, other health outcomes and patient factors that were not tested in this study as the study was not designed to address them, including patient preference for care, patient contextual factors and provider and health system priorities for care..$^{511} 13{ }^{32}$ We also recognize that some of our findings could be due to chance. Future work should assess broader, more patient-oriented longterm outcomes, such as health-related quality of life and mortality and include more patient sociodemographic and context measures.

\section{Implications}

Our findings on the relationship between individual chronic conditions and diabetes care goal achievement have several implications for the design of future multimorbidity interventions if borne out in future research. It is well established that there is a potential to improve control through better self-care and better testing. The importance of BP and LDL control alongside HbAlc control could be emphasized in patient education. Our results support targeted lifestyle interventions for patients with diabetes and co-occurring conditions that are associated with lack of care goal achievement of more than one goal, such as CHF or obesity. Patient-centered medical home elements, such as registries and group visits, ${ }^{36}$ could target patients with 
diabetes and specific comorbidities to provide supportive, integrated diabetes and comorbidity care. ${ }^{26} 37$ This may also help patients and providers prioritize diabetes care goals, such as when achieving a care goal can benefit multiple conditions ${ }^{32}$ and to maximize benefits while decreasing harms, especially among patients in whom it might not be appropriate to achieve all diabetes care goals. ${ }^{7} 38$ Additional previsit planning to ensure fasting laboratory completion, ${ }^{36}$ or non-fasting lipid tests could be used to increase cholesterol testing. ${ }^{39}$ Finally, given that over half of the tested conditions had no relationship with diabetes care, these conditions could be de-emphasized in certain situations in order to create a more manageable list of conditions for which care could be integrated. ${ }^{40}$

Diabetes rarely occurs outside the setting of multimorbidity, multimorbidity complicates diabetes care and interventions are urgently needed to effectively manage these complex patients. Our study shows which conditions are associated with achieving specific diabetes care goals. If these results are borne out in further studies in different populations, future interventions could target patients at risk for not achieving specific diabetes care goals based on their individual comorbidities.

\section{Author affiliations}

${ }^{1}$ Department of Family and Community Medicine, University of California, Davis, Sacramento, California, USA

${ }^{2}$ Health Innovation Program, University of Wisconsin School of Medicine and Public Health, Madison, Wisconsin, USA

${ }^{3}$ Department of Biostatistics and Medical Informatics, University of Wisconsin School of Medicine and Public Health, Madison, Wisconsin, USA

${ }^{4}$ Department of Population Health Sciences, University of Wisconsin School of Medicine and Public Health, Madison, Wisconsin, USA

${ }^{5}$ Department of Medicine, University of Wisconsin School of Medicine and Public Health, Madison, Wisconsin, USA

${ }^{6}$ Department of Family Medicine, University of Wisconsin School of Medicine and Public Health, Madison, Wisconsin, USA

${ }^{7}$ Department of Educational Psychology, University of Wisconsin-Madison, Madison, Wisconsin, USA

${ }^{8}$ Department of Health Informatics and Administration, College of Health Sciences, University Wisconsin Milwaukee, Milwaukee, Wisconsin, USA ${ }^{9}$ Center for Urban Population Health, Milwaukee, Wisconsin, USA

${ }^{10}$ Aurora Research Institute, Aurora Health Care, Milwaukee, Wisconsin, USA

${ }^{11}$ Center for Clinical Epidemiology and Population Health, Marshfield Clinic Research Foundation, Marshfield, WI, USA

${ }^{12}$ Department of Surgery, University of Wisconsin School of Medicine and Public Health, Madison, Wisconsin, USA

Contributors EMM conceptualized the study design, analyzed the data and wrote the manuscript. MP contributed to study design, data analysis and reviewed/edited the manuscript. JEM, NP, DMB, JF and RTG contributed to the study design, results interpretation and reviewed/edited the manuscript. MAS contributed to the study design, data analysis, results interpretation and reviewed/edited the manuscript. EMM serves as the guarantor.

Funding This project was funded by grant R21 HS021899 from the Agency for Healthcare Research and Quality. Additional support was provided by the Health Innovation Program, the UW School of Medicine and Public Health from The Wisconsin Partnership Program, and the Community-Academic Partnerships core of the University of Wisconsin Institute for Clinical and Translational Research (UW ICTR) through the National Center for Advancing Translational Sciences (NCATS), grant UL1TR000427. The work presented here was carried out while Dr Magnan was a Primary Care Research Fellow supported by a National Research Service Award (T32HP10010) from the
Health Resources and Services Administration to the University of Wisconsin Department of Family Medicine. Nancy Pandhi was supported by a National Institute on Aging Mentored Clinical Scientist Research Career Development Award, grant number K08 AG029527.

Competing interests None declared.

Ethics approval University of Wisconsin-Madison.

Provenance and peer review Not commissioned; externally peer reviewed.

Data sharing statement Data sharing is available as per the NIH guidelines.

Open Access This is an Open Access article distributed in accordance with the Creative Commons Attribution Non Commercial (CC BY-NC 4.0) license, which permits others to distribute, remix, adapt, build upon this work noncommercially, and license their derivative works on different terms, provided the original work is properly cited and the use is non-commercial. See: http:// creativecommons.org/licenses/by-nc/4.0/

\section{REFERENCES}

1. Centers for Disease Control and Prevention. National diabetes fact sheet: national estimates and general information on diabetes and prediabetes in the United States, 2011. Atlanta, GA: U.S. Department of Health and Human Services, Centers for Disease Control and Prevention, 2011.

2. Bae S, Rosenthal MB. Patients with multiple chronic conditions do not receive lower quality of preventive care. J Gen Intern Med 2008;23:1933-9.

3. US Department of Health \& Human Services. Multiple chronic conditions - a strategic framework: optimum health and quality of life for individuals with multiple chronic conditions. Washington DC: US Department of Health \& Human Services, 2010.

4. Goodman RA, Boyd C, Tinetti ME, et al. IOM and DHHS meeting on making clinical practice guidelines appropriate for patients with multiple chronic conditions. Ann Fam Med 2014;12:256-9.

5. Bayliss EA, Bonds DE, Boyd CM, et al. Understanding the context of health for persons with multiple chronic conditions: moving from what is the matter to what matters. Ann Fam Med 2014;12:260-9.

6. Uhlig K, Leff B, Kent D, et al. A framework for crafting clinical practice guidelines that are relevant to the care and management of people with multimorbidity. J Gen Intern Med 2014;29:670-9.

7. Smith SM, Soubhi H, Fortin M, et al. Interventions for improving outcomes in patients with multimorbidity in primary care and community settings. Cochrane Database Syst Rev 2012;4:CD006560.

8. Lin EH, Katon W, Von Korff M, et al. Relationship of depression and diabetes self-care, medication adherence, and preventive care. Diabetes Care 2004;27:2154-60.

9. American Diabetes Association. Standards of medical care in diabetes-2013. Diabetes Care 2013;36(Suppl 1):S11-66.

10. Long AN, Dagogo-Jack S. Comorbidities of diabetes and hypertension: mechanisms and approach to target organ protection. J Clin Hypertension (Greenwich) 2011;13:244-51.

11. Piette JD, Kerr EA. The impact of comorbid chronic conditions on diabetes care. Diabetes Care 2006;29:725-31.

12. Magnan $\mathrm{E}$, Palta $\mathrm{M}$, Johnson $\mathrm{H}$, et al. The impact of a patient's concordant and discordant chronic conditions on diabetes care quality measures. J Diabetes Complications 2015;29:288-94.

13. Kerr EA, Heisler M, Krein SL, et al. Beyond comorbidity counts: how do comorbidity type and severity influence diabetes patients' treatment priorities and self-management? J Gen Intern Med 2007;22:1635-40.

14. Pentakota SR, Rajan M, Fincke BG, et al. Does diabetes care differ by type of chronic comorbidity?: an evaluation of the Piette and Kerr framework. Diabetes Care 2012;35:1285-92.

15. Voorham J, Haaijer-Ruskamp FM, Wolffenbuttel BH, et al. Differential effects of comorbidity on antihypertensive and glucose-regulating treatment in diabetes mellitus-a cohort study. PLoS One 2012;7: 38707 .

16. Krein SL, Hofer TP, Holleman R, et al. More than a pain in the neck: how discussing chronic pain affects hypertension medication intensification. J Gen Intern Med 2009;24:911-6.

17. Wisconsin Collaborative for Healthcare Quality. WCHQ ambulatory measure specification: diabetes care performance measures. Middleton, WI: Wisconsin Collaborative for Healthcare Quality, 2011.

18. American Diabetes Association. Standards of medical care in diabetes-2011. Diabetes Care 2011;34(Suppl 1):S11-61.

19. National Committee for Quality Assurance. The State of Health Care Quality 2011: continuous improvement and the expansion of quality 
measurement. Comprehensive Diabetes Care. Washington DC, 2011:50-51.

20. Naessens JM, Stroebel RJ, Finnie DM, et al. Effect of multiple chronic conditions among working-age adults. Am J Manag Care 2011;17:118-22.

21. Hwang W, Weller W, Ireys $\mathrm{H}$, et al. Out-of-pocket medical spending for care of chronic conditions. Health Affairs (Project Hope) 2001;20:267-78.

22. Pham HH, Schrag D, O'Malley AS, et al. Care patterns in Medicare and their implications for pay for performance. $N$ Engl J Med 2007;356:1130-9.

23. University of Wisconsin Department of Population Health. Wisconsin county health rankings. Madison, WI: University of WisconsinMadison, 2006-2014.

24. WWAMI Rural Health Research Center. Rural-Urban Commuting Area Codes (RUCAs). RUCA Data: Code Definitions Version 2.0. Office of Rural Health Policy (ORHP) of the Health Services Administration (HRSA). http://depts.washington.edu/uwruca/rucacodes.php (accessed 30 Jun 2013).

25. Yancy CW, Jessup M, Bozkurt B, et al. 2013 ACCF/AHA guideline for the management of heart failure: a report of the American College of Cardiology Foundation/American Heart Association Task Force on practice guidelines. Circulation 2013;128: e240-327.

26. Zulman DM, Kerr EA, Hofer TP, et al. Patient-provider concordance in the prioritization of health conditions among hypertensive diabetes patients. J Gen Intern Med 2010;25:408-14.

27. Russell LB, Suh DC, Safford MA. Time requirements for diabetes self-management: too much for many? J Fam Pract 2005;54:52-6.

28. Egede LE, Osborn CY. Role of motivation in the relationship between depression, self-care, and glycemic control in adults with type 2 diabetes. Diabetes Educ 2010;36:276-83.
29. Dixon LB, Kreyenbuhl JA, Dickerson FB, et al. A comparison of type 2 diabetes outcomes among persons with and without severe mental illnesses. Psychiatr Serv 2004;55:892-900.

30. Erlich DR, Slawson DC, Shaughnessy A. Diabetes update: long-term treatment of adults. FP Essent 2013;408:14-19.

31. American Diabetes Association. Standards of medical care in diabetes-2014. Diabetes Care 2014;37(Suppl 1):S14-80.

32. Laiteerapong $\mathrm{N}$, Huang $\mathrm{ES}$, Chin $\mathrm{MH}$. Prioritization of care in adults with diabetes and comorbidity. Ann N Y Acad Sci 2011;1243:69-87.

33. National Committee for Quality Assurance. The State of Health Care Quality 2012: focus on obesity and medicare plan improvement, early edition. Comprehensive Diabetes Care. Washington DC, 2012:58-60.

34. Bleich SN, Pickett-Blakely O, Cooper LA. Physician practice patterns of obesity diagnosis and weight-related counseling. Patient Educ Couns 2011;82:123-9.

35. Simon GE, VonKorff M. Recognition, management, and outcomes of depression in primary care. Arch Fam Med 1995;4:99-105.

36. Margolius D, Bodenheimer T. Transforming primary care: from past practice to the practice of the future. Health Affairs (Project Hope) 2010;29:779-84.

37. Duguay C, Gallagher F, Fortin M. The unique experiences of adults with multimorbidity: a phenomenology (poster). J Comorbidity 2014;4:11-21.

38. Wyatt KD, Stuart LM, Brito JP, et al. Out of context: clinical practice guidelines and patients with multiple chronic conditions: a systematic review. Med Care 2014;52(Suppl 3):S92-S100.

39. Sidhu D, Naugler C. Fasting time and lipid levels in a communitybased population: a cross-sectional study. Arch Intern Med 2012;172:1707-10.

40. Zulman DM, Asch SM, Martins SB, et al. Quality of care for patients with multiple chronic conditions: the role of comorbidity interrelatedness. J Gen Intern Med 2014;29:529-37. 